\title{
Enluminures : représentations de William Blake
}

\section{Sarah Wilson}

Traducteur : Agnès Calza et David Lefèvre

\section{OpenEdition \\ Journals}

Édition électronique

URL : https://journals.openedition.org/rbnu/3188

DOI : $10.4000 /$ rbnu.3188

ISSN : 2679-6104

\section{Éditeur}

Bibliothèque nationale et universitaire de Strasbourg

\section{Édition imprimée}

Date de publication : 1 novembre 2011

Pagination : $30-41$

ISSN : 2109-2761

\section{Référence électronique}

Sarah Wilson, "Enluminures : représentations de William Blake », La Revue de la BNU [En ligne], 4| 2011, mis en ligne le 01 novembre 2011, consulté le 08 août 2021. URL : http:// journals.openedition.org/rbnu/3188; DOI : https://doi.org/10.4000/rbnu.3188

\section{(c) (i) (2)(2)}

La Revue de la BNU est mise à disposition selon les termes de la Licence Creative Commons Attribution - Pas d'Utilisation Commerciale - Partage dans les Mêmes Conditions 4.0 International. 


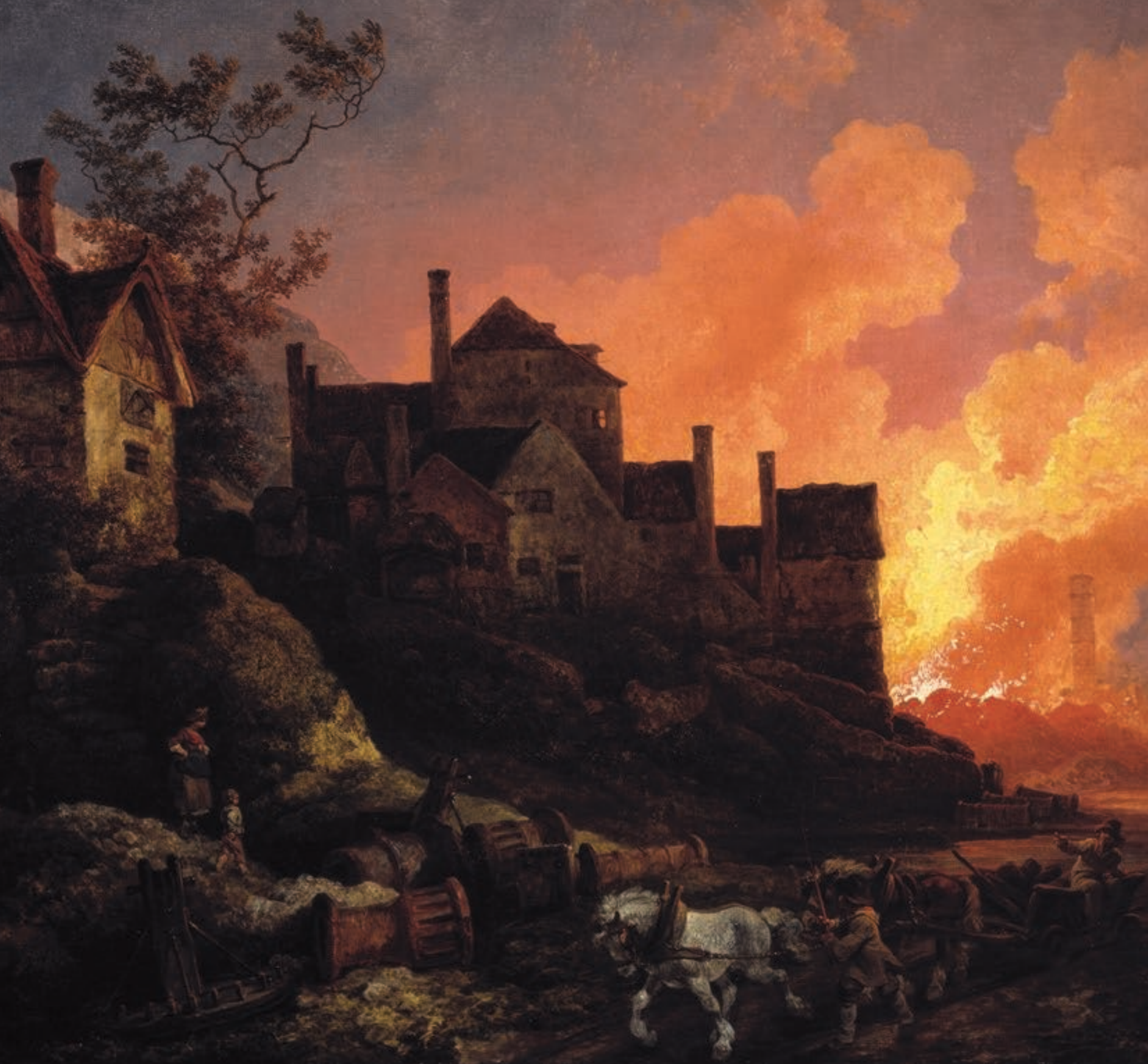




\section{Enluminures : représentations de William Blake}

\section{Pour Mary Jacobus}

Comment imaginer William Blake ? C'est une présence vivante dans nos institutions, nos festivals, notre langue... Sur le parvis de la British Library, à Londres, l'ennemi de Blake, le matérialiste Isaac Newton, est accroupi dans le bronze, le regard fixé sur son compas. La sculpture d'Edoardo Paolozzi, Newton (d'après Blake) de 2002 est devenue paradoxalement le symbole du savoir ${ }^{1}$. Enfant, je chantais " Petit Agneau, qui t’a créé ? Le sais-tu bien, qui t'a fait naître ? " Écolière, j’interprétais avec ma chorale le vibrant et viril appel à l'action de Jerusalem :

Qu'on m'apporte mon arc d'or brûlant ;| Qu'on m'apporte mes flèches de désir ;| Qu'on m'apporte ma lance : ô nuages, ouvrez-vous !| Qu'on m'apporte mon char de feu.

Je ne ferai pas trêve au combat de l'esprit/ Et mon épée ne dormira pas dans ma main/ Tant que nous n'aurons point bâti Jérusalem/ Sur la verte et riante terre d'Angleterre ${ }^{2}$.

(Bring me my Bow of burning gold: Bring me my arrows of desire: Bring me my spear: O clouds unfold, Bring me my chariot of fire!

I will not cease from mental fight Nor shall my sword sleep in my hand: Till we have built Jerusalem In England's green \& pleasant land.)

(traduction de Pierre Leyris in Milton, suivi de Le jugement dernier, éd. Corti, 1999, p. 43) 
Blake évoque ici la croyance ancestrale selon laquelle Joseph d'Arimathie - avec l'enfant Jésus - posa le pied à Glastonbury, où l'on dit que son bâton fleurit3. C'est le sens de la première strophe :

Et ces pieds dans les temps anciens/ Ont-ils foulé les vertes hauteurs de l'Angleterre ?|

Et a-t-on vu le saint Agneau de Dieu/

Sur les riantes pâtures de l'Angleterre?

Et la divine contenance/

A-t-elle resplendi sur nos collines nuageuses ?|

Et Jérusalem était-elle bâtie là/

Parmi ces sombres moulins sataniques ?4

(And did those feet in ancient time

Walk upon England's mountains green?

And was the Holy Lamb of God

On England's pleasant pastures seen!

And did the countenance divine

Shine forth upon our clouded hills?

And was Jerusalem builded here

Among those dark satanic mills?)

(Ibid., p. 43)

L'Angleterre s'appelait aussi Albion ou Britannia ${ }^{5}$. Il s'agissait d'un concept autant militant que mythologique, à l'époque de Blake ${ }^{6}$. Ce dernier est tout entier présent dans Jerusalem. Mystiques et pourtant extravagants, savants et pourtant porteurs d'émotions, ses mots promettent un avenir radieux, reliant passé et présent, temps et espace. Cependant Blake perturbe une langue imprégnée de références bibliques (le " char de feu " est celui d'Ézéchiel) par un anachronisme contemporain choquant : les " sombres moulins sataniques " de la révolution industrielle. Sur la verte Angleterre, Satan œuvre dans les noires cicatrices et les crevasses des usines ${ }^{7}$ et des mines. Le Pandemonium de Milton, qui " s'éleva de la terre comme une exhalaison ", hante cette vision ${ }^{8}$.

Jerusalem est extrait de la préface de Milton, a poem in two books (1804-10). La vision miltonienne de l'enfer de Satan - " point de lumière mais des ténèbres visibles " - est le principe oxymorique qui brille à travers les " illuminations " de Blake. La lumière jail- lit du papier à travers des couleurs sombres ou radieuses ; The Simoniac Pope, de 1794 (tiré de L'Enfer de Dante), traîné en enfer, illustre le mieux ce paradoxe. De la Bible et de Milton, Blake hérite une vision manichéenne à l'oeuvre dans Le mariage du Ciel et de l'Enfer (The Marriage of Heaven and Hell) de 1790-93, et Chansons d'innocence et d'expérience (Songs of Innocence and Experience) de 1789-94. En amont de tout choix d'effet sonore, Blake emploie des procédés qui reflètent ces binômes antagoniques. Le positif et le négatif, la forme et le vide, sont inhérents à son processus d'impression unique. Blake "sculpte " les mots de ses poèmes en relief et à l'envers sur la plaque de cuivre ; il travaille avec l'écriture en tant que reflet d'elle-même. Et son usage de l'illustration comme une sorte de marginalia qui s'étend et dévore la page répond à la fois à la position " marginale " qu'occupent les illustrations par rapport aux tableaux, et aussi à sa propre marginalité - qui contraste avec la mégalomanie de son rôle, auto-proclamé, de barde et de prophète ${ }^{9}$. Formé comme graveur, il donne forme aux plus puissantes des créatures à la plus petite échelle. Un orteil phallique surgit à travers la barbe du patriarche accroupi au début du Premier livre d'Urizen (1794) ${ }^{10}$. La jeune nymphe qui se tient en équilibre dans l'éther à la première page du Livre d'Ahania de 1795 pourrait être vue comme son complément et sa contrepartie féminine.

Alors que nous percevons les vers de Blake sinuant sur la page avec leurs enjambements époustouflants, nous entendons leur pulsation dans notre esprit. La longueur est d'une importance capitale : le besoin de briser les carcans qu'éprouvait Blake fait éclater les pentamètres iambiques de Shakespeare comme le vers plus néoclassique de Milton. Ses rythmes de désir effréné furent repris par Walt Whitman dans Feuilles d'herbes en 1855 et par Allen Ginsberg dans Howl en 1955, exactement un siècle plus tard. En France, ces longs vers, antithèses de l'alexandrin, allaient inspirer Philippe Soupault, Georges Bataille et André Masson pendant la période politique mouvementée de l'entredeux-guerres - suite à la première grande exposition française de Blake en $1947^{11}$.

Les longs vers de Blake s'accumulent pour former les immenses architectures de vers de Visions des filles d'Albion et de L'Amérique, Prophétie, tous deux de 1793 et, à la suite d'Urizen et d'Europe, Prophétie, tous deux de 1794, Le Chant de Los, de 1795, Milton, et sa dernière 


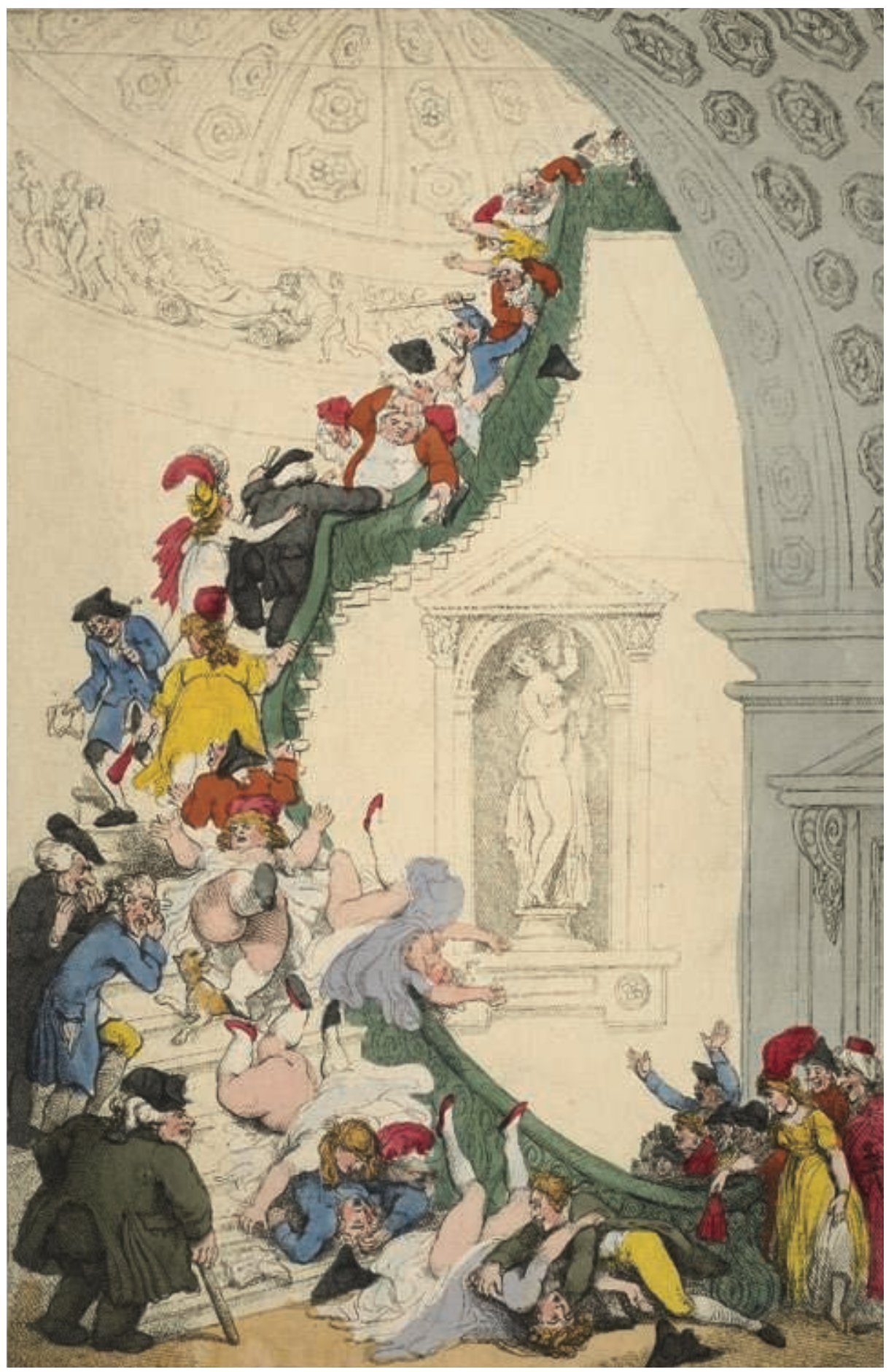

Thomas Rowlandson, Exhibition Stare Case, ca. 1811.

Gravure coloriée à la main, 48.4 x $31.7 \mathrm{~cm}$. (coll. Londres, Trustees of the British Museum) 


\section{PREFACE.}

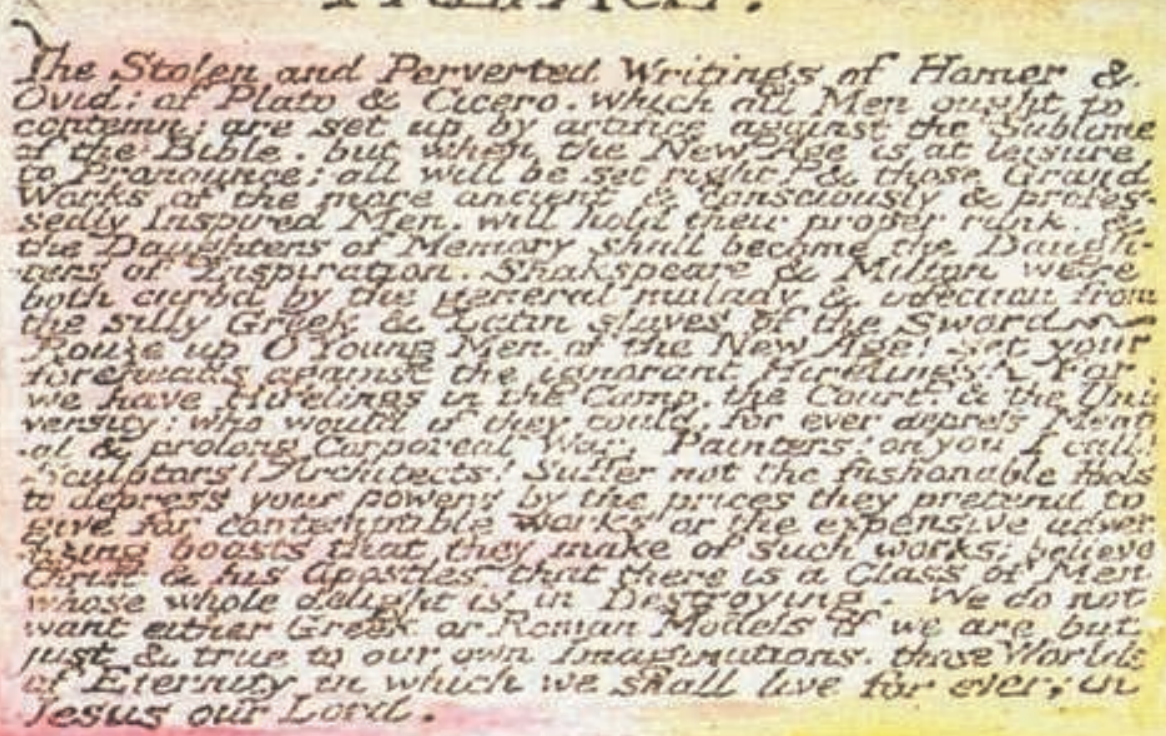

- And did thase feret in uncient time.

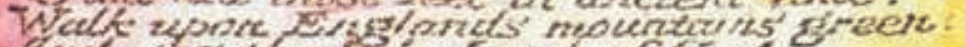
Ared was tre kuly tarze ar God.

on Linglands plecesient pastures seen!

Aud did the Cotmierarce Divize

Shine fainth uport our clorcded rills?

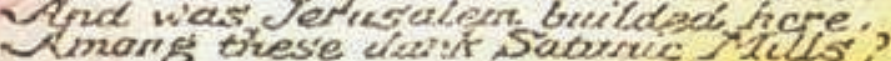
Birves me nu Bow of burring gold: Hivis me nit Spear: O clovels wifbld.

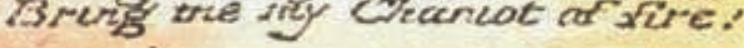

$I$ will not ceuse fram irental Fipht.

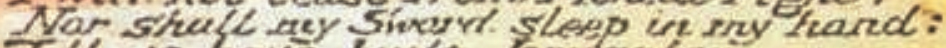
Jull ne have bute berusalerin.

Jit Ainglarels speer \& pleosant Land

Hould to God that all tie tards people were Propkets

Numbers Xr.ch

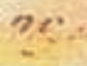

William Blake, Preface (with 'Jerusalem'),

projet abandonné de planche devant suivre la page de titre

de Milton, a Poem, 1804 (ca. 1804-1810).

Gravure aquarellée (coll. New York Public Library) 

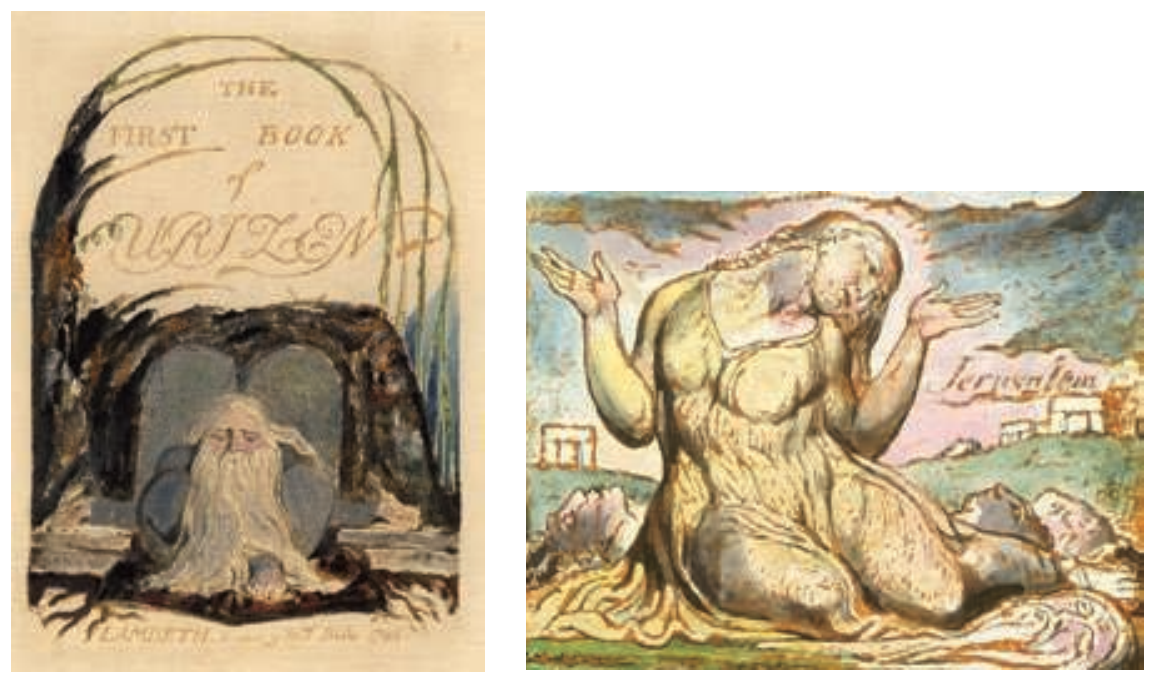

(gauche)

Urizen, 1794. Tiré de A Small Book of Designs,

The First Book of Urizen.

Eau-forte en relief coloriée à la main sur papier, $8.8 \times 10,2 \mathrm{~cm}$. (coll. Londres, Trustees of the British Museum)

(droite)

Enitharmon, tiré de Jerusalem, the emanation of the Giant Albion, 1804-1820, planche 92 (détail). Gravure à l'eau-forte en relief aquarellée, sur papier (coll. Yale Center for British Art, New Haven, Connecticut)

grande œuvre, Jérusalem (à ne pas confondre avec le poème éponyme), écrite de 1804 à 1820. " Jérusalem ", comme l'Angleterre (Stonehenge), figure sur une illustration derrière la voluptueuse Enitharmon, encadrée par la plainte de Los :

Que vois-je! Les Britanniques, Saxons, Romains et Normands fusionnant/

Dans mes fournaises en Une Nation... ${ }^{12}$

La nation pécheresse créée dans nos fournaises

et nos métiers à tisser, c'est Albion...

(What do I see! The British, Saxon, Roman, Norman amalgamating

In my furnaces into One Nation...

This sinful Nation Created in our furnaces and looms is Albion...)

\section{(traduction d'Agnès Calza)}

L'ensemble de l'œuvre de Blake est beaucoup plus vaste. Il semble souvent impossible de démêler sa panoplie de personnages : figures et lieux bibliques, historiques et contemporains s'entrelacent visuellement et mentalement : ainsi dans The Spiritual Form of Nelson guiding Leviathan et The Spiritual Form of Pitt guiding Behemoth ${ }^{13}$. Et pourtant l'éthique de Blake, sa profonde religiosité et son énergie sensuelle transcendent ses " mythèmes ", comme je l'ai jadis compris - aidée par les vivifiantes études d'Harold Bloom sur la transmission de la poésie épique à travers les civilisations ${ }^{14}$.
Contrastant avec l'ampleur et la complexité de Blake, se trouvent ses chansons, avec leur emploi de l'anglais courant. La musique contrapunctique des monosyllabes et des jeux de mots de Blake - le plaintif "weep" (pleurer) dans "sweep" (ramoneur) - demeure intraduisible $^{15}$. Prenons la chanson Joie nouveau-née (Infant joy)

" Je n'ai pas de nom,/

Je n'ai que deux jours »./

Comment t'appellerai-je ?|

" Je suis heureuse :/

Joie est mon nom "./

Aie douce joie en partage !

(I have no name

I am but two days old -

What shall I call thee?

I happy am

Joy is my name -

Sweet joy befall thee! ${ }^{16}$ )

(William Blake, Euvres, vol. 1, trad. Pierre Leyris, Aubier-Flammarion, 1974, p. 201)

Au sein de la vision de Londres qu'avait Blake, vision du dix-huitième siècle vue à travers le prisme de Lambeth, avec ses tendres mères et ses enfants ou ses ramoneurs orphelins en haillons, ses visions apocalyptiques pourraient sembler déplacées ou naïves. Mais Blake, baptisé dans l'Eglise anglicane, bien que connu pour avoir fréquenté l'Eglise de la nouvelle Jérusalem 
de Swedenborg, écrivait à une époque où la Bible du roi Jacques constituait le coeur spirituel de tout foyer ${ }^{17}$. Dans sa lecture quotidienne, sa poésie, ses prophéties, sa portée historique coexistaient avec une pieuse simplicité, une piété simple. Le principe de la topologie, l'Ancien Testament accompli dans le Nouveau, était transféré au temps présent : c'était une Bible à appliquer dans la vie quotidienne ${ }^{18}$. Même le petit enfant, heureux ou exploité, était un concept à la fois mystique et christologique : le Christ représentait à la fois l'innocence, en tant qu'enfant et en accueillant les enfants, et l'expérience, en tant que sage au Temple puis en tant qu'enseignant pour les enfants. Chez Blake, la spiritualité religieuse conjuguée à la vie privée était rendue complexe non seulement par des pratiques swedenborgiennes mais également par des pratiques moraves ésotériques, dont la méditation sexuelle, la visualisation et la sublimation ${ }^{19}$. "Les linéaments du désir comblé " de La réponse à la question trouvent leur apothéose dans Visions des filles d'Albion:

Je crie : Amour ! Amour ! Heureux, heureux amour !| Libre comme le vent des montagnes !

(I cry : Love! Love! Love! Love! Happy, happy Love! Free as the mountain wind $!^{20}$ )

(William Blake, Euvres, vol. 3, trad. Pierre Leyris, Aubier-Flammarion, 1980, p. 215)

La tradition des emblèmes et des livres d'emblèmes - le lien entre les rébus, les images énigmatiques et les maximes religieuses - hante les illustrations de petit format de Blake : ainsi For the Sexes: The Gates of Paradise et There is no natural Religion, qui s'achève par une planche où il est inscrit :

En conséquence,|

Dieu devient semblable|

à ce que nous sommes, afin que nous|

soyons semblables à ce qu'il est.

(Therefore

God becomes as

we are, that we

may become as he ${ }^{21}$ )

(ibid, p. 51)

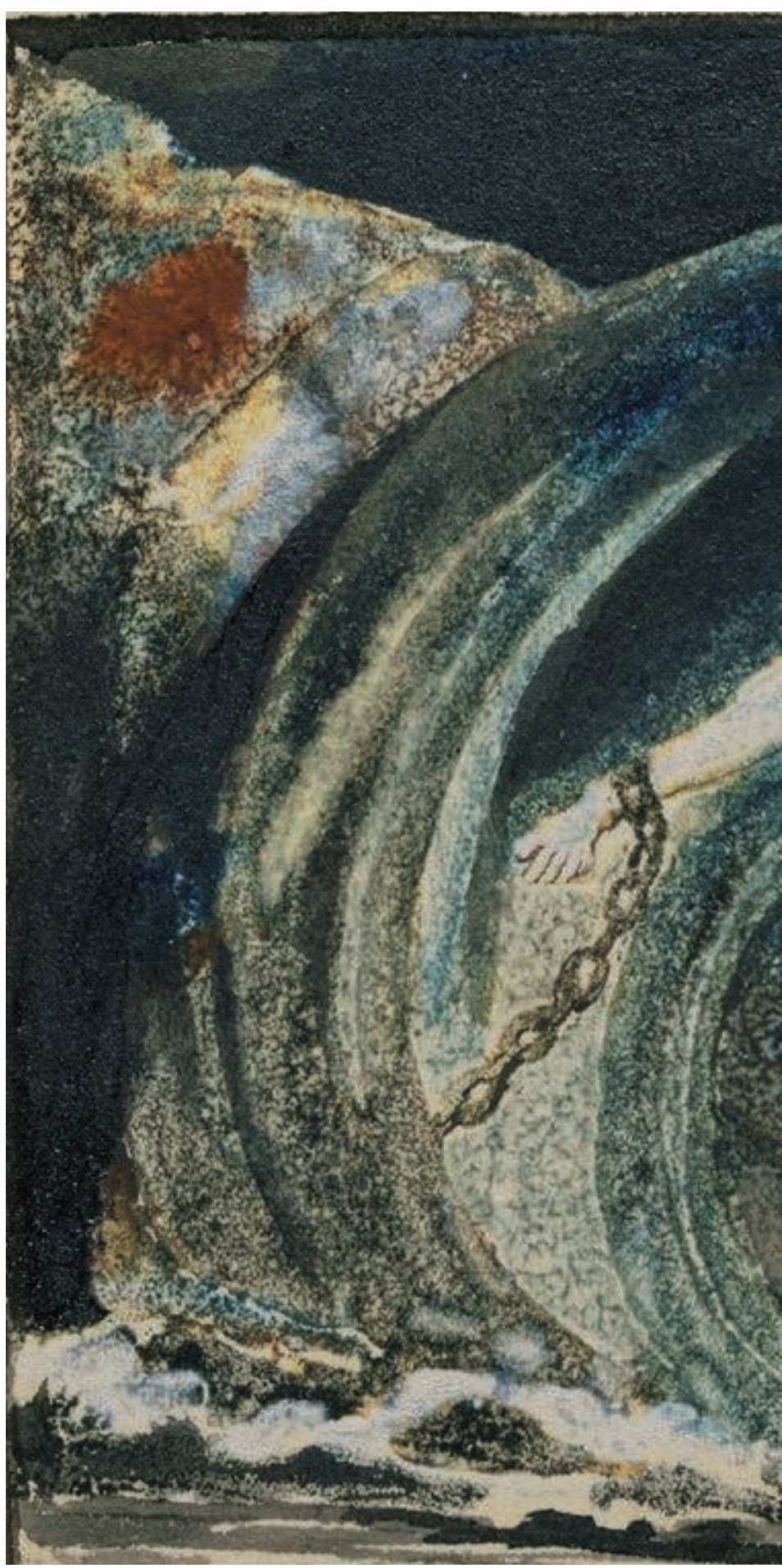




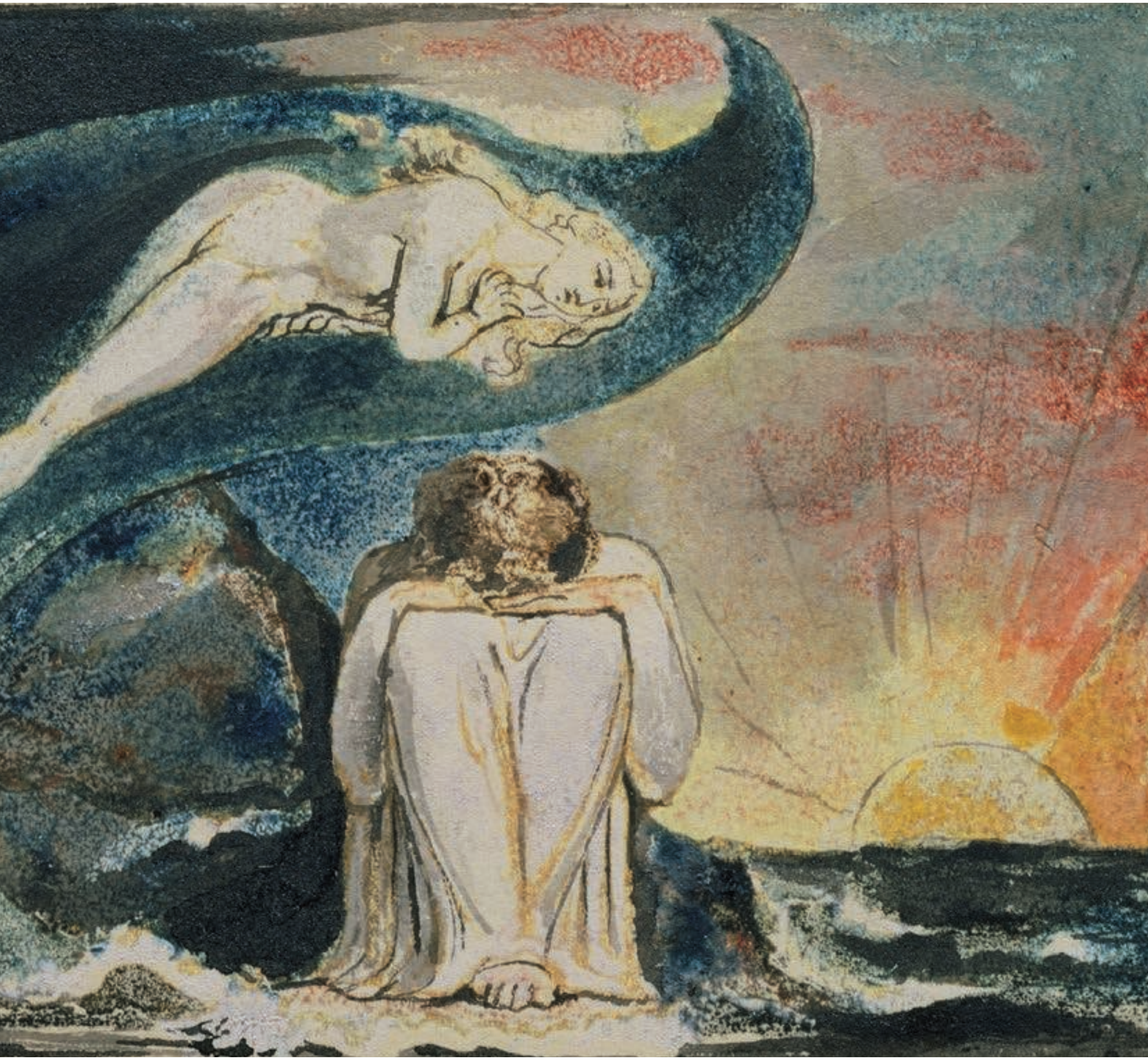

Visions of the Daughters of Albion, ca. 1795. Planche 4.

Eau-forte en relief coloriée à la main sur papier (coll. Tate Gallery, Londres) 
Aucun poète ne peut rivaliser avec la conviction qu'a Blake de communiquer directement avec Dieu ou avec des esprits visionnaires : son propre appel à la prophétie. Il écrivit à John Flaxman en 1800 :

... Milton m'a aimé dans mon enfance et m'a montré son visage./

Ezra est venu avec Isaïa le Prophète, mais Shakespeare dans mes années plus mûres m’a donné la main ;) Paracelse et Boehme me sont apparus, des terreurs sont apparues là-haut dans les Cieux/ Ainsi qu'en bas dans les Enfers, lors un puissant et redoutable changement a menacé la Terre.| La Guerre d'Amérique commença. Toutes mes noires horreurs passèrent devant ma face/ Et traversant l'Atlantique, gagnèrent la France. Alors la Révolution française déferla en d'épaisses nuées,| Et mes anges m'ont dit qu'avec pareilles visions je ne pourrais subsister sur la Terre...

(... Milton lov'd me in childhood \& shew'd me his face. Ezra came with Isaiah the prophet, but Shakespeare in riper years gave me his hand; Paracelsus and Behmen appear'd to me terrors appear'd in the heavens above And in Hell beneath, \& a mighty \& awful change threatened the Earth.

The American War began. All its dark horrors passed before my face

Across the Atlantic to France. Then the French Revolution commenc'd in thick clouds,

And My Angels have told me that seeing such visions I could not subsist on earth....2)

(Ecrits prophétiques des dernières années, suivis de Lettres, éd. José Corti, 2000, p. 131)

Blake rejoint ainsi une tradition de mystiques anglais, issus du Petit livre des révelations (Revelations of Divine Love) de Julienne de Norwich, où la langue aspire à la transcription visionnaire plutôt qu'à l'invention littéraire. Imaginons aussi la joie éprouvée par Blake dans la musique : le jeune artiste faisant des croquis dans l'abbaye de Westminster alors que ses hautes voûtes gothiques résonnent des liturgies chantées et des sonorités chorales de l'époque. Thomas Tallis ou William Byrd étaient alors supplantés par des compositeurs modernes comme Georg Friedrich Haendel, coqueluche de la société londonienne, fondateur de la Royal Academy of Music en 1719. Son Messie (Messiah) de 1741, aussi populaire que le Jérusalem de Blake, prend sa place au sein d'une tradition anglaise profondément liée à l'année liturgique dans nos cathédrales et salles de concert.

La religiosité de Blake coexistait néanmoins avec un désir ardent de libération des systèmes, des institutions, et des " menottes forgées par l'esprit " (Londres dans Chants d'innocence et d'expérience). Il faisait partie $\mathrm{du}$ milieu intellectuel gravitant autour de Thomas Paine (Les droits de l'homme - Rights of Man, de 1791), Mary Wollstonecraft (Défense des droits de la femme - A Vindicaton of the Rights of Woman, de 1792) et son mari William Godwin (An Enquiry concerning Political Justice, de 1793) ${ }^{23}$, inédit en France. Contrastant avec sa lecture de Wisdom of Angels $(1788,1790)$ et Heaven and Hell (1790) de Swedenborg (tout deux inédits en France), il désapprouvait le sublime d'Edmund Burke, l'Essai sur l'entendement humain - Essay on Human Understanding de Locke, Voltaire et Rousseau. Blake était un fin connaisseur de la science de son temps (il illustra les essais de Lavater sur la physiognomonie). Sa plus grande hostilité personnelle concernait le doyen de la Royal Academy, Sir Joshua Reynolds. Ses annotations aux Discours (Discourses) de Reynolds ont été décrites comme " un intellectualisme de guérilla, vulgaire et incisif, iconoclaste et scandaleux $»^{24}$.

Franchissant le seuil de Somerset House, dans le Strand de Londres, je pénètre dans la Royal Academy of Arts de Blake ${ }^{25}$. Je monte l'escalier en colimaçon et en encorbellement dont se moqua le caricaturiste Thomas Rowlandson en 1800. Stare Case, sa gravure en couleurs émoustillante, montre des dames dégringolant les marches en un amas bruyant. Les " connaisseurs " reluquaient leurs derrières et leurs jupons : l'Académie était l'endroit où courtiser le plus chic de Londres $^{26}$. Fondée longtemps après la Royal Academy of Music, en 1768, elle s'installa dans le nouveau bâtiment de William Chambers en 1780, demeure de "Sir Joshua et la bande de fripons à sa solde ${ }^{27}$. C'était le centre du monde de l'art londonien. Contrairement à Blake, ou Hogarth, Reynolds avait effectué l'obligatoire voyage à Rome ; c'était le président d'une structure hiérarchique remontant, au-delà de son modèle immédiat, le Salon de Paris, jusqu'à l'Italie elle-même. $\mathrm{Au}$ sommet de l'escalier, dans le Grand Salon de Somerset House, The Tribune of the Uffizi (1780) de Johann Zoffany, incluant une vingtaine de portraits de cognos- 
centi anglais, était reconstituée dans la peinture britannique contemporaine, tandis qu'un public varié regardait à la fois les murs recouverts de tableaux et se regardait lui-même ${ }^{28}$. Le portrait de Reynolds, H.R.H. George, Prince of Wales (1787), insufflait au présent ostentatoire des prétentions Renaissance (dans le portrait, le serviteur noir du Prince Régent suscitait des ricanements : le mouvement abolitionniste débutait à Londres cette année-là).

On y trouvait des scènes historiques et mythologiques (Didon de Reynolds, Samson de John Francis Rigaud) et des portraits d'aristocrates ou d'actrices comme la Giovanna Bacelli (1782) de Gainsborough. Turner et Constable bouleversaient la tradition de la peinture de paysage ; on y trouvait de la peinture de genre, de la sculpture, de l'aquarelle et de la gravure. John Flaxman et l'extravagant Henry Fuseli étaient des amis intimes de Blake (Nightmare de Fuseli fut également exposé en 1782 ; lui aussi était adepte de sujets shakespeariens et miltoniens). Dans les écoles de l'Académie et à la bibliothèque, Raphaël et Michel-Ange, les héros musclés de Blake, étaient glorifiés. C'étaient les privilèges, le mercantilisme et les bavardages vulgaires dans le Grand Salon à propos du " goût " qui le dégoûtaient ${ }^{29}$.

Le fossé - et les rapports économiques - entre ce monde et les " sombres moulins sataniques " de Jérusalem ne pouvaient être plus frappants. Pourtant à l'Académie, en 1801, Philippe-Jacques de Loutherbourg le Jeune exposait A View of Colebrook Dale by night. Ici, les hauts fourneaux à charbon illuminent le sombre paysage du Shropshire. Le ciel rougeoyant évoque les représentations de Sodome et Gomorrhe par les primitifs flamands et anticipe les apocalypses victoriennes de John Martin $^{30}$. Colebrook Dale, en tant que sujet " moderne ", industriel, est ramené dans le passé par le contexte moral implicite : les prochaines décennies l'abandonneraient en hâte dans la course aux marchés et au profit ${ }^{31}$. Blake illustra son poème Jérusalem trois ans seulement après l'exposition de Colebrooke Dale. Quand John Martin réalisait son propre Sodom and Gomorrah en 1852, La situation de la classe ouvrière en Angleterre en 1844 de Friedrich Engels avait été publiée en Allemagne ${ }^{32}$. Engels à Manchester et Karl Marx dans la Londres du milieu de l'époque victorienne allaient élargir les prémices dialectiques de Blake, avec un impact révolutionnaire bien plus important que celui de $1789 . .$. Blake, dédaigné de tous de son vivant, à l'exception de quelques rares élus, mourut comme un mystique ésotérique, presque inconnu.

Pourtant, en opposition à sa redécouverte par les poètes symbolistes Swinburne et W.B. Yeats, un Blake repositionné dans son siècle industriel allait émerger ${ }^{33}$. Aux heures les plus sombres de l'histoire de Londres, tandis que Jacob Bronowski, né à Lodz, travaillait sur les stratégies du Bomber Command, il écrivait sur Blake, reliant l'artiste à Marx, et les débuts de l'industrialisation au développement de la "guerre totale ${ }^{34}$. Le poète surréaliste Humphrey Jennings, inspiré par les rythmes et la vision flamboyante de Blake, décrivit la cathédrale St Paul durant le Blitz dans I see London. Il cita Jérusalem dans son film Words for Battle, en 1941, et dressa son propre Pandaemonium : 1660-1886 The Coming of the Machine Age as Seen by Contemporary Observers ${ }^{35}$.

Pour en revenir à Jérusalem et à mes désirs d'écolière : "Qu'on m'apporte mon Arc d'or brûlant!" Quand Charles Hubert Parry mit en musique les mots de Blake, nous étions en 1916 : un moment tragique précédant la guerre totale. Parry était vigilant quant à son instrumentalisation - jusqu'à ce que le mouvement des suffragettes demande à utiliser sa chanson $^{36}$. Étrangement Jérusalem, dans toute sa noblesse ou sa sensiblerie, a toujours appartenu aux femmes ${ }^{37}$. Ainsi, pourrait-on dire, en était-il de Blake : d'abord à sa mère, Catherine Blake, puis à sa femme et collaboratrice, Catherine Sophie Boucher ${ }^{38}$. Le Blake dont les mythologies m'ont déconcertée plus tard à Oxford " appartenait " à Northrop Frye et à Harold Bloom. A la lecture de Visions des filles d'Albion nos réactions 
nous paraissaient étrangement transgressives dans un univers blakien encore patriarcal ${ }^{39}$. Aujourd'hui, Blake l'ésotérique a été profondément féminisé. Nous savons " Pourquoi pleurait Mme Blake" ("Why Mrs Blake cried "), nous comprenons l'aspect " queer " de la jouissance chez Blake, ainsi que les théories swedenborgiennes de l'amour conjugal révélées dans Vala ou les Quatre vivants. Les chercheurs femmes et les blakiens les plus "queer " nous invitent à l'aborder de manière plus impertinente ${ }^{40}$. Dans ce monde fantastique, Blake rencontre Tracey Emin de la Royal Academy. L'espace féminin est un espace qu'elle crée et partage avec celui décrit par Blake : l'espace créé par "Enitharmon afin de contenir Satan... pour contenir le désastre $»^{41}$ :

La nature d'un espace féminin est la suivante :

elle rapetisse/

Les organes de la vie jusqu'à ce qu'ils deviennent finis et que lui-même semble infini.

(The nature of a Female Space is this: it shrinks the Organs

Of Life till they become Finite \& Itself seems Infinite ${ }^{42}$ )

(Milton, suivi de Le Jugement Dernier, éd. José Corti, 1999, p. 69)

Georges Bataille conclut son étude sur Blake avec le grand pet céleste de Nobodaddy, son grand juron, avant d'inciter Blake à l'action ${ }^{43}$. La fameuse tente d'Emin, son lit, ses " relations merdiques " perpétuent une tradition. La généalogie qui va de Swedenborg à Blake et Sade, à Hölderlin et à Nietzsche, de Van Gogh à Bataille et à Artaud est la généalogie d'une Europe des esprits. En se tournant vers le siècle des Lumières français, sa brillance et ses certitudes, nous apercevons aussi, comme Blake, la Terreur ${ }^{44}$. De Blake, Mary Wollstonecraft et l'Emma Hamilton de Nelson à Ginsburg et au Blake étrange et "queer " d'aujourd'hui, nous pouvons retracer une transmission alternative d'énergies ${ }^{45}$. L'Angleterre divisée de la Jérusalem de Blake, toute de vert et de noir, avec ses moulins sataniques, son snobisme, sa vulgarité et son bellicisme - ou les pleurs d'un enfant pauvre de Londres - est parmi nous à présent. Blake est toujours contemporain.

\section{Notes}

1 - Les Philosophiae naturalis principa mathematica de Newton (1687) cristallisaient une vision du monde rationaliste et mécaniste que Blake détestait.

2 - Le poème de Blake, dans la préface illustrée de Milton, figure comme planche additionnelle $\mathrm{n}^{\circ} 1$ dans William Blake : the complete illuminated books / David Bindman éd., Londres, Thames and Hudson, 2000, p. 295 Blake renonça à cette page !

3 - Joseph of Arimathea among the rocks of Albion fut la première gravure signée par Blake en 1773 (à l'âge de 16 ans). Glastonbury, le plus ancien sanctuaire chrétien britannique, est aujourd'hui encore un lieu de célébrations païennes et de festivals de musique. Voir " The Jerusalem big hug on Glastonbury Tor at summer solstice, 2010 " : http://www.youtube.com/wat ch?v=iNyb2iDILOI\&feature=related. Il est primordial de distinguer l'usage historique d' " Angleterre ", " Anglais " et " Bretagne » de l'usage habituel de " britannique » (la nationalité).

4- Http://en.wikipedia.org/wiki/And_did_those_feet_in_ancient_time explique parfaitement l'aspect mythologique, musicologique et l'utilisation ultérieure de ce poème !

5- Voir le " Glossaire " d'Armand Himy in William Blake, poète et peintre, Fayard, 2008, p. 311 : «ALBION : nom du géant primitif qui conquit l'Angleterre et lui légua son nom (...) Albion symbolise aussi tour à tour l'Angleterre, l'Adam Kadmon de la Cabale, l'homme moderne déchu. Son sommeil et son réveil illustrent la déchéance puis la renaissance spirituelle de l'homme ou de l'Angleterre " (Himy ne fait aucune référence au poème Jerusalem).

6 - Voir Jacob Bronowski, William Blake and the age of revolution, London, Routledge and Kegan Paul, 1972 (1965, écrit dès 1942) p. 23 (non traduit en français) : « Car le folklore récent d'une Albion druidique avait aussi été utilisé par ceux qui combattaient la cour de George III avec plus de robustesse... les petits hommes... considéraient les anciens Britanniques comme les ancêtres de leurs corporations ".

7 - NdT : « mills » signifie « moulins » mais aussi « usines ».

8 - Le paradis perdu / John Milton; traduction de Chateaubriand, Paris : Renault, 1861 (Livre 1)

9 - Voir les annotations de Blake aux Discourses de Sir Joshua Reynold (1798), cop. 1808 dans l'édition de Geoffrey Keynes de Blake, Complete writings [1966, revue en 1972], 1974, pp. 445-479 et la contribution de Darby Lewes, "Marginal(ized) Blake : the annotations to Reynold's Discourses ", in Lewes ed., Double vision. Literary palimpsests of the eighteenth and nineteenth centuries, Plymouth, Lexington books, 2008, pp. 15-26. J'utilise le mot " dévore " en référence au commentaire de Michael Farrell sur « la synesthésie anthropomorphique " ("vent affamé » - " hungy wind », " rivages colériques " - " angry shores "), p. 33.

10 -Pour une explication sur l'« orteil phallique ", voir Marsha Keith Schuchard, Why Mrs. Blake cried, London, Pimlico, 2007, p. 286.

11- Philippe Soupault vit les œuvres de Blake à la Tate Gallery en 1914 ; voir 'William Blake' (1927) in Écrits sur la peinture, Paris, Lachenal et Ritter, 1980, pp. 85-130 ; Georges Bataille, 'William Blake ou la vérité du mal' in Critique, 1948, pp. 83-107 et William Blake, traduit et présenté par Georges Bataille, dessins d'André Masson, Saint-Clément-de-Rivière, Fata morgana, 2008. L'exposition à la Galerie René Drouin en 1947 fut capitale.

12-Blake, Jérusalem, planche 92 (Keynes, 1974, p. 739 ; Bindman, 2000, p. 389)

13-En référence à l'amiral Nelson (représenté nu) et au premier ministre William Pitt. Ces peintures a tempera (Tate) furent les deux premières $œ u v r e s$ décrites dans 'Advertisements of a descriptive catalogue' (Keynes, 1974, pp. 564-565) publié à l'occasion de l'exposition indépendante de Blake en 1809 .

\section{Sarah Wilson}

(traduction Agnès Calza et David Lefèvre) 
14-C'était lors de la publication retentissante des deux ouvrages de Harold Bloom : The ringers in the tower. Studies in romantic tradition (Chicago, University of Chicago press, 1971) et The anxiety of influence (New York, Oxford university press, 1973)

15- Himy, 2008, p. 75

16-Infant joy in Songs of innocence (Keynes, 1974, p. 118 ; Bindman, 2000, p. 67)

17- La Grande-Bretagne fête cette année le $400^{\mathrm{e}}$ anniversaire de la publication de la Bible du roi Jacques (1611)

18- Voir l'étude remarquable de Christopher Rowland, Blake and the Bible, London and New Jersey, Yale University press, 2010

19- Ces questions sont au cœur de la nouvelle étude publiée dans Why Mrs. Blake cried, Schuchard, 2007. Elle raconte son baptême (à St James, Piccadilly) et qu'en 1749 l'Église morave fut reconnue en tant qu'alliée épiscopale des Anglicans.

20- The Question Answr'd, Poems from the notebook, 1793 ; Visions of the daughters of Albion, planche 7 (Keynes, 1974, pp. 180, 194)

21-For the sexes : the gates of paradise, 1793 et There is no natural religion, 1794; pour le poème, voir Bindman, 2000, p. 30 et 41 (version de 1795). Voir Piloo Nanavutty, 'Blake and emblem literature', Journal of the Courtauld and Warburg Institutes, vol. 15, 3-4, 1952, pp. 258-261

22- Lettre à Flaxman, 12 septembre 1800 (Keynes, 1974, p. 799)

23- Blake illustra Original stories from real life de Wollstonecraft (London, J. Johnson, 1791) (en français Marie et Caroline, ou Entretiens d'une institutrice avec ses élèves, traduit de l'anglais par Marie Vollstonecraft Godwin. Paris, Dentu, 1799)

24- Lewes, 2008, p. 24.

25- Le Courtauld Institute of art a son siège à Somerset House depuis 1989 ; le Courtauld Institute of art occupe l'aile nord, jadis Royal Academy of art ; l'Académie a emménagé dans son emplacement actuel, Burlington House, en 1867 .

26- Voir 'Stare cases : engendering the Public's Two Bodies at the Royal Academy of arts', K. Dina Kriz, in David H. Solkin éd., Art on the line, the Royal Academy exhibitions at Somerset House, 1780-1836, London, Yale University press, 2001, p. 55 ff.

27- Blake, annotations to Reynolds, 1798 (Keynes, 1974, p. 445)

28 - Voir Edward Dayes, Somerset House, London, 1788, aquarelle, collection Spooner, Courtault Institute Galleries, London, tranchant avec le Zoffany décrit par Solkin, voir ci-dessous.

29- Voir Solkin, "The Great Mart of Genius " : the Royal Academy exhibitions at Somerset House, $1780-1836$ in Solkin ed., Art on the line, op. cit. (61381 visiteurs en 1780,91827 en 1822 , p. 5) et, du même auteur, Painting for money. The visual art and the public sphere in eighteenth-century England, Yale, New Haven and London, 1993

30- Comparer les deux œuvres de Martin Sodom and Gomorrah, 1852 et The great day of his wrath, 1853 ; voir John Martin, Tate Britain, 2011

31- Le lieu peint par De Loutherbourg est aujourd'hui un site patrimonial dirigé par les Ironbridge Gorge Museums.

32- Friedrich Engels, History of the working classes in England in 1844 (Die Lager der arbeitenden Klasse in England, Leipzig, 1845; en anglais : New York, 1887), London, Sonnenschein, 1892. Pour Blake en tant que précurseur de Marx à Londres, voir Bronowski, 1972, pp. 181, 190-191

33-Voir Algernon Charles Swinburne, William Blake : a critical essay, London, John Camden Hotten, 1868 ; W. B. Yeats, Ideas of good and evil, London and Dublin, Bullen, 1903, pp. 168-225 ; Arthur Symons, William Blake, London, A. Constable, 1907

34-Voir Bronowski, 1972 ; passages sur la 'guerre totale’ p. 15 ; Blake et Marx pp. 190-191. Le cercle de Cambridge des années 1930 auquel appartenait Bronowski avait aussi pour membres Humphrey Jennings et la poétesse Kathleen Raine (William Blake, London, Thames and Hudson, 1970)

35-Mari-Lou Jennings et Charles Madge éds., Pandaemonium : 1660-1886 The coming of age as seen by contemporary observers, compilé par Humphrey Jennings, London, Deutsch, 1985
36- Jerusalem vit le jour en tant que poème dans une anthologie patriotique de 1916 ; le poète lauréat Robert Bridges en commanda à Parry une adaptation pour l'orgue pour une campagne de combat civique ('Fight for right'); atterré par l' "ultra-patriotisme", Parry fut ravi de l'orchestrer pour un concert de démonstration des suffragettes en mars 1918. Il fut adopté par le Women's Institute en tant qu'hymne pour le vote des femmes.

37- La vidéoJerusalem par 'Saphistische' est conseillée sur You Tube en référence à l'interprétation de Jerusalem au Women's Institute dans le film de Nigel Cole Calendar girls avec Helen Mirren ; voir http://www.youtube. com/watch?v=9r81ZPHfKXU

38- On voit bien l'importance du rôle de sa mère et de sa femme dans Schuchard, 2007

39- Jonathan Wordsworth, descendant de William, dirigea nos lectures à Exeter college, Oxford, avec Mary Jacobus (voir la dédicace). J’ai préféré ma propre copie de Keynes, 1974, à l'archive électronique http://www. blakearchive.org/blake

40-Voir Schuchard, Why Mrs. Blake cried, 2007 ; Helen P. Bruder éd., Women reading Blake, Bruder and Tristane Conolly éds., Queer Blake, les deux publiés à Basingstoke, Palgrave Macmillan, 2007 et 2010

41- Tristane Connolly, 'Fear not/ To unfold your dark visions of torment' : Blake and Emin's bad sex aesthetic ", ibid., pp. 116-139

42- Blake, Milton, 10 (Keynes, 1974, p. 49o) in Connolly, ibid., p. 136

43- Blake, 'When Klopstock England defied...' (Keynes, 1974, p. 186). Voir Bataille, 1957, p. 160 ; 'nobody (personne)... daddy (papa)... dérisoirement Dieu le Père.

Car le vieux Nobodaddy là-haut

Péta, rota et toussa ;

Puis il sortit un grand juron qui fit trembler la terre,

Et appela l'Anglais Blake à grands cris.

44-Voir aussi Bertrand Astorg, Introduction au monde de la Terreur. De SaintJust, Sade et Blake à Ernst Jünger, Paris, Seuil, 1945

45- 'Weird', de l'ancien anglais wyrd ('sort, destin'), de 'celui qui vient' 